\title{
Smectic layer instabilities in liquid crystals
}

\author{
Ingo Dierking*1 ${ }^{*}$ Michel Mitov ${ }^{2}$, Mikhail A. Osipov ${ }^{3}$ \\ ${ }^{1}$ School of Physics and Astronomy, University of Manchester, Schuster Building, Oxford \\ Road, Manchester M13 9PL, United Kingdom \\ ingo.dierking@manchester.ac.uk \\ ${ }^{2}$ Centre d'Elaboration de Matériaux et d'Etudes Structurales (CEMES), CNRS, \\ BP94347, 29 rue Jeanne-Marvig, F-31055 Toulouse cedex 4, France \\ ${ }^{3}$ Department of Mathematics, University of Strathclyde, 26 Richmond Street, Glasgow \\ G1 1XH, United Kingdom
}

Key words: liquid crystal, polymer-stabilized liquid crystal, smectic, ferroelectricity, layer rotation, layer instability, horizontal chevron 


\begin{abstract}
Scientists aspire to understand the underlying physics behind the formation of instabilities in soft matter and how to manipulate them for diverse investigations, while engineers aim to design materials that inhibit or impede the nucleation and growth of these instabilities in critical applications. The present paper reviews the field-induced rotational instabilities which may occur in chiral smectic liquid-crystalline layers when subjected to an asymmetric electric field. Such instabilities destroy the so-named bookshelf geometry (in which the smectic layers are normal to the cell surfaces) and have a detrimental effect on all applications of ferroelectric liquid crystals as optical materials. The transformation of the bookshelf geometry into horizontal chevron structures (in which each layer is in a V-shaped structure), and the reorientation dynamics of these chevrons, are discussed in details with respect to the electric field conditions, the material properties and the boundary conditions. Particular attention is given to the polymerstabilisation of smectic phases as a way to forbid the occurrence of instabilities and the decline of related electro-optical performances. It is also shown which benefit may be gained from layer instabilities to enhance the alignment of the liquid-crystalline geometry in practical devices, such as optical recording by ferroelectric liquid crystals. Finally, the theoretical background of layer instabilities is given and discussed in relation to the experimental data.
\end{abstract}




\section{Introduction}

Liquid crystals were discovered in 1888 by the botanist Friedrich Reinitzer, who unexpectedly observed "two melting points" for crystals extracted from the root of a carrot. The recognition of these mesomorphic states of matter by the scientific community required more than two decades [1]. Today, liquid crystals [2-7] are commonly known by their applications as materials used in displays [8,9]. But liquid crystals are much more than materials employed in image display; they also pave the way to switchable adaptive and nonlinear optics, addressable waveguides and sensor materials [10]. At the same time, liquid crystals are ideal materials to study a wide range of fundamental phenomena in soft matter physics and material science [11,12], such as phase transitions, chirality [13], ferroelectricity in fluids [14,15], pattern formation [16], colloidal and polymer composites [17-21] or defect structures and dynamics [22,23], and living matter [24-27].

\subsection{The self-organized order of liquid crystals}

Liquid crystals are partially ordered fluids which are thermodynamically stable between the (isotropic) liquid and the (three-dimensionally ordered) crystal (fig. 1). They combine the anisotropic properties of the crystalline phase with the positional disorder of molecules in a liquid. Numerous individual liquid crystal phases are distinguished. We

will here limit our discussion to thermotropic liquid crystals, which are observed purely on change of temperature. Lyotropic phases [28], i.e. those exhibited by a change of 
concentration of an appropriate solvent, are not covered in this review. The simplest thermotropic liquid crystal phase is the nematic, which exhibits only orientational order of the long axis of rod-like (calamitic) molecules. The two phases that this review is mainly focused on are the chiral fluid smectic phases $\mathrm{SmA}^{*}$ and $\mathrm{SmC}^{*}$. In addition to orientational order of the long molecular axis along a preferred direction called the director $\mathbf{n}$, these phases also exhibit a one dimensional positional order, with molecules arranged in a layered structure, which may realistically better be described as a one dimensional density distribution. In the (achiral) smectic A phase the director n, which also represents the optic axis of the phase, is oriented parallel to the smectic layer normal $\mathbf{k}$, while in the (achiral) SmC phase the director is inclined to the layer normal by a temperature dependent angle $\theta$, called the tilt angle. The respective structural features are summarised in fig. 1.

\subsection{Ferroelectricity in chiral (fluid) smectic liquid crystals}

The introduction of chirality into liquid crystals, either by making the mesogens themselves chiral or by adding chiral dopant molecules to an achiral host, leads to a wealth of novel phenomena [29], such as the formation of helical superstructures, the observation of novel phases and polar effects, such as ferroelectricity or the electroclinic effect, which is similar to the piezoelectric effect in solid state materials.

Meyer et al. [30] first demonstrated on the basis of symmetry considerations that every

tilted chiral smectic phase can exhibit a spontaneous polarisation $\mathbf{P}_{\mathbf{S}}$, i.e. exhibit pyroelectricity. The achiral $\mathrm{SmC}$ phase is of $\mathrm{C}_{2 \mathrm{~h}}$ symmetry and shows the symmetry 
elements of figure 2: (i) a two-fold rotation axis $\mathrm{C}_{2 \mathrm{y}}$, (ii) a mirror plane $\mathrm{m}_{\mathrm{xz}}$ and thus (iii) an inversion centre i. Applying inversion symmetry to an arbitrary permanent electric dipole moment results in a vanishing spontaneous polarization. The situation is different when the smectic $\mathrm{C}$ phase is composed of chiral molecules. In this case the mirror plane vanishes, and so does the inversion centre, while the two-fold rotation axis is retained. The $\mathrm{SmC}^{*}$ phase is of $\mathrm{C}_{2}$ symmetry, and applying the only remaining symmetry element to a general dipole moment leads to a non-zero spontaneous polarization, $\mathbf{P}_{\mathbf{S}}=\mathrm{P}_{0}(\mathbf{k} \times \mathbf{n})$. Introduction of chirality also leads to the observation of the electroclinic effect $[31,32]$, a deviation of the director from its equilibrium orientation by application of an electric field. The deviation occurs perpendicular to the electric field direction and is linear in field amplitude, i.e. also dependent on polarity. The electroclinic effect is strongly nonlinear with respect to temperature, diverging at the $\mathrm{SmA}^{*}-\mathrm{SmC}^{*}$ phase transition. It is present in both phases, but can be most easily observed in the SmA* phase, as here it is not obscured by the ferroelectric switching of the $\mathrm{SmC}^{*}$ phase. The electroclinic effect causes an induced tilt angle $\theta_{\text {ind }}$ :

$\theta_{\text {ind }}=\frac{e E}{\alpha\left(T-T_{C}\right)}$

where e is the material dependent electroclinic coefficient, E the electric field amplitude, and $\alpha\left(T-T_{C}\right)$ the coefficient of the first term in the Landau expansion of the free energy density.

Within the context of smectic layer instabilities, both ferroelectricity and electroclinic effect, i.e. spontaneous and induced polarisations in chiral materials are of fundamental importance. None of the behaviour reported below has been observed in achiral liquid crystals. 


\section{Smectic $\mathrm{C}^{*}$ layer and director configurations}

The $\mathrm{SmC}^{*}$ structure exhibits a surprising wealth of different director and smectic layer configurations, depending on chirality, boundary conditions and other applied external forces. The fundamental phenomena observed are shortly summarised below.

\subsection{The helical bulk state}

In the bulk state the spontaneous polarisation of the $\mathrm{SmC}^{*}$ phase is cancelled on a microscopic scale, due to the formation of a helical superstructure [33], as depicted in fig. 3(a). When advancing along the direction of the smectic layer normal, the director processes on a cone with a temperature dependent opening angle of twice the tilt angle, 20. Since the spontaneous polarisation is perpendicular to the director and lies in the smectic layer plane, $\mathbf{P}_{\mathbf{S}}$ cancels over the spatial period of one pitch of the helical superstructure. The pitch of the $\mathrm{SmC}^{*}$ phase generally ranges from $100 \mathrm{~nm}$ to several tens of micrometers. If it is in the range of several micrometers, a periodic line pattern can easily be observed in polarising microscopy due to the helical superstructure (fig. 3(b)), and the bulk SmC* phase should thus rather be called helielectric [34]. The direction of the line pattern is equivalent to the smectic layer plane, which is useful to determine the smectic layer orientation. 


\subsection{Surface influences on smectic layer geometries}

Clark and Lagerwall [35] demonstrated that if a SmC* phase is confined to very thin cells with the gap approximately smaller than the pitch of the helix, the latter is unwound by surface interactions, resulting in a ferroelectric domain structure. This geometry is called the surface stabilised ferroelectric liquid crystal (SSFLC) state. In the ideal case the smectic layers are oriented perpendicular to the bounding substrates, in the so called bookshelf geometry schematically shown in fig. 4(a). This allows switching of the spontaneous polarisation from up to down orientation, which is accompanied by a director reorientation of twice the tilt angle on the cone, giving maximum contrast of the device for appropriately oriented crossed polarisers. The respective zero field virgin ferroelectric domain structure of the bookshelf geometry is depicted in fig. 4(b).

Generally, the situation is somewhat more complicated with vertical chevron structures being formed. These have been intensively studied by Rieker and co-workers [36-38], but also by Ouchi and coworkers [39]. Due to smectic layer contraction under the constraint of parallel anchoring when passing the $\mathrm{SmA}^{*}-\mathrm{SmC}^{*}$ transition, smectic layers in the $\mathrm{SmC}^{*}$ phase tilt with respect to the substrate normal, as schematically shown in fig. 5(a). The layer tilt angle is often similar to the director tilt angle, $\delta \sim \theta$, and tilt can occur in both directions. This leads to defects called zig-zags [39], where regions of opposite layer tilt meet. Thin defect lines are of the type $\langle\langle<>>>$, while thick lines are of type $>>><<<$. Chevron structures decrease device contrast and viewing angle characteristics (fig. 5(b)). A texture example of zig-zag defects is shown in fig. 5(c). 


\section{Electric field effects on smectic layers}

\subsection{Smectic layer straightening}

It had long been thought that once formed, smectic layer structures are fixed. This view was revised through $\mathrm{x}$-ray [40-42] as well as electro-optic [43] investigations, which indicated a reversible smectic $\mathrm{C}^{*}$ layer straightening under application of an electric field in device geometry. Detailed time resolved synchrotron studies [44-46] confirmed the proposed behaviour of a reversibly decreasing chevron angle under applied electric fields of relatively small amplitude. The investigations also indicated that the direction of the smectic layer normal may change in the plane of the cell substrate. The reason for smectic layer straightening lies in the fact that in a vertical chevron geometry the spontaneous polarisation is not parallel to the applied electric field vector, because the smectic layers are tilted with respect to the substrate plane (fig. 5(a)). This exerts a torque on the smectic layers trying to straighten them in order to orient the spontaneous polarisation parallel to the applied electric field.

\subsection{The horizontal chevron formation process}

While for moderate electric field amplitudes the smectic layer straightening is a reversible process, for large amplitudes it can induce an irreversible rearrangement of smectic layers to form what has become known as 'horizontal chevrons', a term first coined by Jakli and Saupe [47]. The process of reorienting a vertical chevron structure 
(fig. 5(b)) into a bookshelf structure (fig. 4(a)) by application of large electric fields may in principle be achieved via two possible mechanisms: (i) smectic layers irreversibly straighten with the smectic layer normal $\mathbf{k}$ being parallel to the rubbing direction $\mathbf{x}$. This implies that two bookshelf domains are being formed with director orientations of $n= \pm \theta$ tilted with respect to the smectic layer normal $\mathbf{k}$ and thus also the rubbing direction $\mathbf{x}$. (ii) the smectic layers reorient such that the layer normal $\mathbf{k}$ tilts with respect to the rubbing direction $\mathbf{x}$ by $\mathrm{k}= \pm \theta$, while the director $\mathbf{n}$ in both ferroelectric domains points along the rubbing direction $\mathbf{x}$.

As the substrate boundary conditions generally promote molecular alignment along the rubbing direction, the scenario most often observed is that of scheme (ii). During the irreversible smectic layer straightening process, a vertical chevron structure is transformed into a horizontal chevron structure.

The striped bookshelf texture of the $\mathrm{SmA}^{*}$ phase $[22,48]$ is one example of such a resultant smectic layer reorientation structure, with stripes basically oriented along the rubbing direction. Figures 6(a) and (b) depict the schematic director and smectic layer configuration, and a typical texture photograph, respectively. Such structures can be induced by application of AC electric fields, preferably to thin cells [49-52], and their periodicity is approximately equal to the employed cell gap $[47,50,53]$. The angle formed between the smectic layer normal $\mathbf{k}$ and the rubbing direction $\mathbf{x}$ is generally smaller than the induced director tilt angle $\theta$ [54].

The observed periodicity, as well as the small layer inclination angles distinguish the striped bookshelf layer arrangement of the SmA* phase from horizontal chevron domains of the SmC* phase. The latter exhibit no periodicity, but nevertheless often elongated 
domains due to the influences of the rubbing direction. But via which process are horizontal chevrons actually formed?

One first has to realise that the zig-zag regions of vertical chevron structures are actually of the bookshelf-type, but with the layer normal inclined to the rubbing direction (fig 7(a)). As the smectic layers straighten for increasing electric field amplitude, these bookshelf-zones increase at the expense of the vertical chevron until the two domains of oppositely inclined bookshelf domains meet. The defect wall mediating between these two domains is of the vertical chevron type [55] (fig. 7(b)). The smectic layer structure can easily be verified when viewing a helical sample between crossed polarisers at zero applied electric field condition (fig. 7(c)), where the helix lines indicate that the smectic layers are inclined with respect to the rubbing direction, in opposite directions for the two respective domain types. It should also be noted that the horizontal chevron domain formation is not related to any surface memory effects, as domains of different shape and position are formed for consecutive experiments [56]. The horizontal chevron domain structure can thus be erased by heating into the isotropic phase.

For samples with a suppressed helical superstructure, the polarisation up and polarisation down domains appear equivalent between crossed polarisers, mediated by a bright defect line. Application of a positive field switches both domains into the polarisation up state where one domain stays black, while the other switches to its bright state. Polarity reversal leads to a reversal of dark and bright domains. This behaviour is demonstrated in fig. 8, together with a schematic of the smectic layer structure and the director orientations. The smectic layer structures can also be elucidated by small angle x-ray 
investigations on oriented samples (fig. 8(c)), giving evidence for the discussed structures [57].

Already the textures of figure 8 imply a detrimental effect of the formation of horizontal chevrons on the electro-optic performance of any device, as both the polarisation up and down state consists of an equal distribution of dark and bright domains. Thus, no significant electro-optic response is observed on electric field polarity inversion, as demonstrated in fig. 9. While each individual domain exhibits the well known bistable electro-optic response of the a ferroelectric liquid crystal, although phase shifted by $180^{\circ}$ for both domain types, the overall optic response of the horizontal chevron domain device tends to zero, independent of the position of the device between crossed polarisers.

The dynamics of the formation process of horizontal chevron domains is discussed in detail in ref. [56] and summarised in fig. 10. For increasing electric field amplitude, the time for the formation of horizontal chevrons strongly decreases, while the average domain width increases until saturation (fig. 10(a)). Increasing the frequency of the applied electric field results in an increase of the time of formation with the domains formed exhibiting somewhat small average domain sizes (fig. 10(b)). The reduced temperature below the $\mathrm{SmA}^{*}$-SmC* transition only has a pronounced effect on domain formation dynamics and size in the close vicinity of the transition with faster domain formation of larger size (fig. 10(c)). It is presumed that the latter behaviour is related to a strong softening of the smectic layer structure, as it is generally observed that the smectic layer compressibility is largely enhance at phase transitions from the cholesteric phase $[58,59]$ 


\section{Smectic layer rotational instabilities}

An effect not often talked about, possibly also due to its detrimental influences on the electro-optic properties of FLC devices, is the so called smectic layer rotation or smectic layer rotational instabilities. Application of asymmetric electric waveforms, as they are often used in addressing schemes, may lead to an in-plane rotation of smectic layers in mainly the $\mathrm{SmC}^{*}$ phase, but due to the electroclinic effect also in the $\mathrm{SmA} *$ phase. This effect had first been reported by the Gothenburg group at the 13th International Liquid Crystal Conference Vancouver, Canada [60] and was later elaborated on at other conferences $[61,62]$. A rotation of the smectic layer normal in the plane of the substrate by several hundreds of degrees had been reported for the application of a sawtooth field to a high electroclinic material in the $\mathrm{SmA}^{*}$ phase. Although strikingly beautiful, this work [60-62] was never published and fig. 11 depicts a texture example of their observed spiral instabilities after rotation of the smectic layers for several tens of minutes and hundreds of degrees. First reports of the smectic layer rotation in literature were published by the Osaka group on electroclinic $\operatorname{SmA}^{*}[63]$, ferroelectric $\operatorname{SmC}^{*}[64,65]$, and antiferroelectric $\mathrm{SmC}_{\mathrm{A}}$ * phases [66,67], which will be discussed in more detail below.

\subsection{Dependence of the dynamics on external parameters}

At first it should be noted that the in-plane rotation of smectic layers can be induced by any type of asymmetric electric waveforms. These can be amplitude asymmetric or time 
asymmetric without DC balance, time asymmetric with DC balance, or any combination thereof [68]. There has also been one report of smectic layer rotation by a pure DC field [69], although it is not quite clear what constitutes the mechanism of this rotation, which could possibly also be related to ionic contamination. Secondly, the layer rotation can be continuous for weak anchoring conditions, or limited to an angle of approximately twice the tilt angle (in fact twice the horizontal chevron inclination [55]) for strong anchoring [70,71]. Thirdly, it is only observed for chiral materials (see also below).

In the following we will examine the dependence of the rotation of smectic layers for strong anchoring conditions. A rotation time, $t_{\mathrm{r}}$, can be define which corresponds to the time needed to rotate a virgin horizontal chevron structure, obtained by application of sufficiently large electric fields into one, where all smectic layers are inclined to the same direction. For an applied electric field with DC bias, but qualitatively also for all other applied asymmetric fields, it is found that the rotation time exponentially decreases for increasing field amplitude, as depicted in fig. 12(a). The frequency dependence of the rotation time is somewhat more complicated and depends on the applied bias (fig. 12(b)). For small bias the rotation times increase and exhibit a maximum at approximately $100 \mathrm{~Hz}$, the typical relaxation frequency of ions in a liquid crystal, before they decrease due to the faster director switching and vanishing of the ionic influences. Above the saturation frequency at about $5 \mathrm{kHz}$, director switching slowly ceases, and the layer rotation times increase again. For larger bias fields the ions quickly get trapped at the electrodes, and the rotation process becomes faster with increasing frequency, again until the director switching slowly ceases for several $\mathrm{kHz}$. Approaching the $\mathrm{SmC}^{*}-\mathrm{SmA}^{*}$ transition temperature from below, the rotation times decrease with increasing 
temperature, as shown in fig. 12(c). It is presumed that this is the effect of two mechanisms, firstly a decrease in viscosity, which implies a faster layer rotation process and secondly, the softening of the smectic layers (decrease in layer compression modulus). It is anticipated that a similar effect would be observed for de Vries ferroelectrics, because the smectic layers are already "soft", as only a tilt ordering is needed to achieve the transition from $\mathrm{SmA}^{*}$ to $\mathrm{SmC}^{*}$. At last, the rotation time decreases with increasing sandwich cell gap, which can be attributed to the vanishing influence of the boundary conditions for the bulk of the liquid crystal as the cell gap is increased (fig. 12(d)).

The latter behaviour hints towards the mechanism of the smectic layer rotational instability [72], which is a permeation process of molecules across smectic layers. This can microscopically be observed through the motion of domain boundaries and deformations of formerly round air bubble enclosures toward an elongated shape. Applying an asymmetric electric field to a horizontal chevron structure causes molecules of the unfavoured domain orientation to permeate in the direction of rubbing, this translation takes place primarily at the domain wall to the favoured domain, thus causing a domain wall motion roughly in the direction perpendicular to the rubbing direction (see fig. 13(a)). This can be illustrated by the textures with helix lines, which indicate the smectic layer direction (fig. 13(b)), and is evidenced by small angle x-ray diffraction (fig. 13(c)).

Starting with a horizontal chevron structure, application of an asymmetric electric field causes a layer rotation until the sample is uniformly inclined with the layer normal making an angle with the rubbing direction equal to the director tilt angle. Reversal of the 
asymmetry causes a rotation in the opposite direction (through nucleation and domain growth) until the layer normal is now oppositely inclined to the rubbing direction. From the x-ray diagrams one can also see that during the rotation the smectic layer order parameter decreases, and increases again after the rotation process is completed. Note that to accomplish the whole reorientation process, molecules only need to translate over distances comparable to their molecular length.

\subsection{Dependence of the dynamics on other parameters}

The smectic layer rotational instability does not only depend on applied external conditions, but also on several parameters of the system itself. In the following, these will shortly be outlined and discussed. It was stressed above that the observed layer rotation only occurs in chiral materials. This can also be inferred by investigations of mixtures of the two left and right handed enantiomers of a FLC compound [73], as depicted in fig. 14(a). The general trend of a decreasing layer rotation time for increasing asymmetry ratio of an applied electric field is clearly observed for all concentrations. For the pure enantiomers with enantiomeric excess ee $=-1$ and $e e=+1$ the same rotation times are observed, only that the domains rotate in opposite direction. This is also the fastest reorientation process. As the enantiomeric excess becomes smaller and tends toward zero, ee $\rightarrow 0$, the rotation time increases, seen to diverge exactly at ee $=0$ [74]. This clearly implies that chirality is needed to observe the smectic layer rotation.

Fig. 14(b) shows the asymmetry ratio dependence of the layer rotation times for several different alignment layers [75]. Quite a variation of rotation times is observed, which can 
be attributed to varying anchoring strengths of the substrates, increasing from polyimide used in commercial EHC cells, via polyimide from Dupont to Nylon 6/6. It should be noted that similar results are obtained when using the same substrate coating material and varying the number of rubbing cycles at constant pressure, or varying the rubbing pressure at a constant number of cycles. The anchoring strength increases with the number of rubbing cycles, as well as rubbing pressure. In both cases an increase of the smectic layer rotation times is observed.

Already the frequency dependence of the layer rotation times suggests a certain influence of ions on the speed of the rotation process. To study this influence, one can deliberately contaminate the liquid crystal through doping with an ionic dopant [76], as it normally used for dynamic light scattering displays. The results are depicted in fig. 14(c) and it is clear that the rotation time decreases as the ionic dopant concentration increases. A possible explanation of this behaviour could be electroconvective effects in the domain walls separating domains of opposite layer inclination. This would increasingly facilitate the permeation flow of molecules across adjacent smectic layers, decreasing the rotation time as the ionic concentration increases.

At last, the domain rotation time also depends on the nature of the phase transition (fig. 14(d)), as shown for a homologous series of ferroelectric liquid crystals with largely varying phase sequences $[73,77]$. The shorter homologues exhibit a $\mathrm{N}^{*}$-SmC* transition (among a range of other transitions), for which the quickest rotation process is observed in the smectic $\mathrm{C}^{*}$ phase. This seems to be independent of the molecular length. For a $\mathrm{SmA}^{*}-\mathrm{SmC} *$ transition, clearly longer domain rotation times are observed at the same reduced temperature in the $\mathrm{SmC}^{*}$ phase. The smectic layer rotation process is 
significantly slower, especially when considering the doubling of the applied field amplitude. The medium length homologue D7 exhibits a phase sequence which incorporates a narrow TGBA* twist grain boundary phase. Here the rotation times are of intermediate magnitude, again if equivalent reduced temperatures in the smectic $\mathrm{C}^{*}$ phase are considered. It is anticipated that this behaviour is due to the softening of the smectic layers, which appears to be larger approaching a $\mathrm{N}^{*}$ phase than a SmA* phase. This is intuitively understandable, as the $\mathrm{SmA}^{*}$ phase is a fluid smectic phase, just as $\mathrm{SmC}^{*}$, while in the $\mathrm{N}^{*}$ phase all positional ordering vanishes. With the TGBA* phase being somewhat similar to $\mathrm{N}^{*}$, but additionally also exhibiting local smectic ordering, one would expect values to lie in between those of $\mathrm{N}^{*}$ and $\mathrm{SmA}^{*}$, as observed.

\section{Applicational aspects}

Despite its detrimental influence on the electro optic performance, the smectic smectic layer reorientation can nevertheless be employed for the benefit of ferroelectric liquid crystal devices. Some of these beneficial properties are outlined below, for example to obtain well aligned smectic layer configurations, possibilities for display damage recovery, or applications such as erasable optical recording, or simple display devices which only need voltages applied during image change, for example as switchable supermarket price tags. 


\subsection{Uniform alignment and texture damage recovery}

A straight forward application of the formation process of horizontal chevrons in combination with the smectic layer reorientation is found for the generation of uniform alignment of ferroelectric liquid crystals [78]. As outlined above, smectic $\mathrm{C}^{*}$ phases, when formed from their non-tilted smectic $\mathrm{A}^{*}$ high temperature phase, most often exhibit (vertical) chevrons, which decrease the electrooptic performance, for example viewing angle and contrast ratio. Applying an electric field with sufficient amplitude changes the vertical into a horizontal domain structure. Subsequent application of an asymmetric AC field rotates all smectic layers into a uniform configuration with the smectic layer normal inclined to the rubbing direction by the amount of the tilt angle. Fixture of the crossed polarizers in an appropriate way the leads to a dark position for one voltage and a bright position for reversed polarity of the field.

Similarly, this procedure can be applied for display damage recovery. For nematic liquid crystals the uniformity of the director alignment is self-healing, or through applying pressure to the screen. This is not the case for smectic liquid crystals. Nevertheless the healing process of smectic layers can be aided by heating the device into the isotropic

phase, then cooling it into smectic $\mathrm{C}^{*}$, and applying the above outlined procedure.

\subsection{Simple low power displays}

Using the smectic layer reorientation, simple displays can be envisioned which only need an applied voltage during changes made to the black and white image (on, off pixel). 
These could be employed for example in electronic price tags for supermarket products. The method of operation is straight forward. Switching between bright and dark is not via the normal director switching of an FLC, but rather through the reorientation of the smectic layers from one to the other inclination by applying electric fields of opposite asymmetry. Obviously, this will not be a high definition display, rather similar in information content to that used for wrist watches or pocket calculators, and also changing the respective information displayed will take seconds to minutes, but, unlike a TN display, no power is required to hold the information.

\subsection{Optical recording}

The applicability to employ the smectic layer reorientation in devices for optical recording has been demonstrated by the Yoshino group [79-82]. It can be used with materials of different phase sequence, namely $\mathrm{N}^{*}-\mathrm{SmC}^{*}$ [79] and SmA*-SmC* $[80,81]$. In the former case the writing and erasure process is as follows: on cooling from the $\mathrm{N}^{*}$ phase, one of the two possible smectic layer arrangements can be selected by selection of the asymmetry of the applied electric field. Erasure can be realised by heating the whole cell into the $\mathrm{N}^{*}$ phase, which can simply be done via temperature control, but also locally by laser heating, or photoisomerization of a suitable dopant molecule. This allows for two layer arrangements, corresponding to a binary image. In the latter case, also grey-levels can be achieved. The recording process uses the fact that the layer rotation is much faster in the $\mathrm{SmC}^{*}$ than in the $\mathrm{SmA}^{*}$ phase. The cell is kept in the ferroelectric phase at temperatures below the $\mathrm{SmC}^{*}-\mathrm{SmA}^{*}$ transition. Parts of the device are irradiated by a 
laser and heated into the $\mathrm{SmA}^{*}$ phase. In the irradiated area no layer rotation will be observed, while in the non-irradiated areas the smectic layers can be rotated to any desired position, depending on the duration of asymmetric voltage application. The laser is then turned off and the whole sample reverts back to the $\mathrm{SmC}^{*}$ phase. This optical recording process is schematically shown in fig. 15(a) and demonstrated in fig 15(b). Erasure of the pattern can be achieved by a reversal of the writing process outlined above. The recorded pattern is stable against application of symmetric electric fields, and more complicated patterns can be designed [82].

\section{Polymer-stabilized smectic layer structures}

Since the smectic layer instabilities generally exhibit a detrimental effect on the electrooptic properties of a ferroelectric liquid crystal, ways may be designed to prohibit their occurrence all together. One of such ways is the so-named polymer-stabilization, which is based on the formation of a porous polymer network inside the volume of a liquid crystal, while largely maintaining the electro-optic properties. This approach is discussed in the following paragraphs.

\subsection{Polymer-stabilized liquid crystals: main features}

Polymer-stabilized LCs (PSLCs) [17-19] are composite materials which are obtained by dissolving a small amount of a bifunctional photo-reactive mesogenic monomers (typically between 1 and $10 \mathrm{wt} . \%$ ) in a low molar mass LC, together with a 
photo-initiator. The gap of a sandwich-cell is filled with the mixture and the thin film is oriented in the desired director configuration. The polymer-network forming material is polymerized and crosslinked by UV light irradiation. After polymerization, the LC is trapped in the network which follows the mesophase order and acts as a template of the original texture, as it has been demonstrated by optical microscopy, scanning (or transmission) electron microscopy for a variety of mesophases [17-19,83-85]. PSLCs have stimulated reflection on fundamental insights - such as LC-polymer interactions, polymer percolation in an anisotropic fluid, template properties, phase transitions under random confinement - and novel potential applications like mechanically stabilized FLC displays [86,87], reflective displays $[9,88,89]$, heat repelling sheets or smart windows [90] .

\subsection{Suppression of smectic layer reorientation}

Rotational instabilities may appear in textures of chiral smectic LCs when subjected to an asymmetric electric field as a consequence of smectic layer reorientations, as discussed above. This undesirable phenomenon can be prevented by the curing of a few per-cent of photopolymerizable monomers dispersed in the LC [91-93]. In addition to stabilizing the smectic layer order, the upper stability of the LC order is considerably raised. By introduction of a polymer network, a template of the pristine director configuration is created, i.e. a memory state, now exerting a mechanical field on the liquid crystal. The velocity of layer reorientation is decreased when increasing the polymer network content, until the complete suppression of instabilities occurs. Optical 
microscopy textures do not drastically change after the polymerization process, which indicates that the network consists of smooth strands causing only small inhomogenities to the LC orientation (fig. 16). Anisotropic patterns (stripe texture) are stored well above the clearing temperature of the liquid crystal before polymerization, due to molecules following the order of the template. Electro-optic properties are not drastically altered [19]. In the $\mathrm{SmA}^{*}$ phase, there is no significant change in the induced tilt angle behaviour or magnitude upon polymerization, while the rise time is increased after curing. In the SmC* phase, the tilt angle is slightly increased and also the spontaneous polarization, while the rise time is not significantly increased (thus still being compatible with display technology requirements). Only if the polymerization is carried out in the isotropic phase, the polymer has no imparted order and prevents any bulk alignment, which affects the electro-optic characteristics.

The network acts in two ways: first it gives a biased elastic torque counteracting a field of such symmetry as to cause a change from the templated layer direction; second, it introduces an increased effective viscosity counteracting the reorientation in both directions [94]. The smectic layer reorientation is strongly hindered by intra-layer polymerized networks as compared to inter-layer polymerization, which is in accordance with the above discussed permeation explanation of the layer reorientation. For the intralayer polymerized samples with a polymer network formed basically perpendicular to the rubbing direction (at small layer tilt angles), the mass flow in a direction of rubbing is strongly hindered, whereas it is permitted for the polymer network formed along the smectic layer normal $[94,95]$. 


\subsection{Polymer network influence on FLC parameters}

The degree of elastic interaction between LC and polymer largely governs the electro-optic response of PSFLCs. The Landau-de Gennes potential incorporating an elastic coupling term, permits to describe the influence of a phase separated polymer network on the experimental properties of a FLC [96]. The elastic coupling between the network and the LC director increases linearly with increasing polymer content in PSFLCs cured in the SmA* phase. The network gives a contribution of $10-15 \%$ of the total free energy density [97].

With low concentrations of polymer, optical response and tilt angle remain fairly independent of concentration but as the concentration increases, switching speed and tilt angle may decrease dramatically [98]. By polymerizing at particular temperatures, characteristics of the PSFLC approach those exhibited by the FLC [99]. The rate of polymerization increases significantly as the order of the mesophase increases and the temperature decreases. The impact of the polymerization temperature on the electro-optic properties strongly depends on the choice of monomer $[99,100]$. Both the ferroelectric polarization and the optical response time increase with polymerization temperature in the ordered phases. FLC properties such as spontaneous polarization, threshold field and switching speed depend on the polymerization kinetics. Ferroelectric properties are less affected when the UV light irradiation conditions correspond to a low power and a long irradiation time $[101,102]$. The network may facilitate the so called V-shaped switching and shorten the response time [103]. A network formed at large tilt angle well into the $\mathrm{SmC}^{*}$ phase tries to preserve the $\mathrm{SmC}^{*}$ director orientation as the $\mathrm{SmC}^{*}-\mathrm{SmA} A^{*}$ 
transition is approached whereas polymerization in the $\mathrm{SmA} *$ phase results in a reduction of the polarization [104]. The dielectric strength at the $\mathrm{SmC}^{*}$ to $\mathrm{SmA}^{*}$ transition increase with increasing polymer concentration until saturation, and so do the relaxation frequencies in the $\mathrm{SmC}^{*}$ phase. The Goldstone-mode within the $\mathrm{SmC}^{*}$ phase is suppressed when the polymerization is carried out in the SmA* phase [105]. The longterm stability of the quasi-bookshelf texture is significantly improved as well as the contrast and multiplex addressing performances, by relieving the stress at the stripe interfaces [106].

Polymer stabilisation to increase mechanical stability of related devices retains the superior electro-optic properties of $\mathrm{SmC}^{*}$ and $\mathrm{SmA}^{*}$ phases, like very fast response times, little dependence on deviations from normal light incidence, high contrast or the ability for easy linear greyscale generation, or light modulation. The dynamic range of devices may even be increased through polymer stabilization [107].

\section{Theory of smectic layer reorientation}

\subsection{Simple symmetry arguments}

It is possible to describe qualitatively the layer rotation induced by the external electric field using some very generals symmetry arguments. Indeed, let us consider the $\mathrm{SmA}$ layer with an electric field $\mathbf{E}$ applied parallel to the smectic plane. In this configuration the electric field specifies a polar direction in the plane of the smectic layer, and the axis of the layer rotation is expected to be parallel to this direction, which corresponds to the experiment. This is not surprising, as the electric field is the only vectorial physical 
quantity of the system. The rotation is characterized by the angular velocity $\omega$ and for symmetry reasons it is expected to be parallel to the electric field $\mathbf{E}$.

However, the relationship between the external electric field and the angular velocity of layer rotation is not completely specified before the time reversal symmetry is also taken into consideration. One notes that the angular velocity $\omega$ changes sign under time reversal while the electric field does not. Thus in the linear approximation the angular velocity $\omega$ should be proportional to the time derivative of the electric field E which also changes sign under time reversal:

$$
\omega=\alpha \dot{E}
$$

One notes that in this simple equation the angular velocity $\omega$ is the so called pseudovector which does not change sign under space inversion while the electric field as well as its time derivative are polar vectors which do change sign under space inversion. This contradiction can be resolved if one assumes that the coefficient $\alpha$ is a pseudo-scalar and therefore the medium must be chiral. Then the product $\alpha \dot{E}$ on the right hand side of eq. (1) is also a pseudo-vector which has the same transformation properties as $\omega$. This conclusion again corresponds with existing experimental data which indicate that the layer rotation is observed only in chiral smectic phases.

Thus, from a general symmetry point of view, Eq.(1) indicates that the rotation of chiral smectic layers in a time-dependent electric field is allowed by symmetry. The angular velocity of the layer rotation must change sign under time reversal of the external field, which indeed is observed experimentally. 
It should be noted that the rotation is not expected to occur in a constant field which appears to be in contradiction with the experiment [69Error! Reference source not found.] where layer rotation in the $\mathrm{SmC}^{*}$ phase has been observed in a DC electric field. One notes, however, that in this experiment the liquid crystal was doped by charged impurities which produced an electric current across the cell. Taking into account that the symmetry of electric current $\mathbf{I}$ is exactly the same as that of the time derivative of the electric field one can write down a similar linear relationship between the angular velocity and the electric current

$$
\omega=\alpha \mathbf{I}
$$

Thus it also follows from symmetry consideration that the rotation of chiral smectic layers can be induced by an electric current.

Equations $(1,2)$ are very general but they do not contain any information about a particular mechanism of rotation in chiral smectic phases. Such a mechanism for the $\mathrm{SmA}^{*}$ phase is discussed in detail below. However, before considering the mechanism of the rotation, let us first investigate the consequences of these general equations.

One notes that in all experiments the electric field $\mathbf{E}(t)$ is periodic and thus the time derivative $\dot{\mathrm{E}}$ changes signs during the period. According to Eq.(1) this means that the layers are rotating in opposite directions for positive and negative values of $\dot{\mathrm{E}}$, respectively. The period of the electric field is usually very small, and thus only the constant part of the angular velocity is actually observed i.e., the zero-frequency component of $\omega$. This zero frequency component is the average angular velocity $\omega_{0}$ which can be obtained by integration of Eq.(1) over time 


$$
\omega=\int_{0}^{\tau} \alpha \dot{E}_{d t}
$$

where $t$ is the period of the field. One can readily see that for any periodic field the integral in Eq.(2) vanishes, and thus, on average, there is no rotation.

However, one should also take into account that the orientation of smectic layers is always stabilized by some counter-torque, which stems from an interactions with the bounding glass plates. Thus the smectic layers are actually rotating only if the driving torque exceeds some threshold value that is during the part of the period when the time derivative $\dot{\mathrm{E}}$ is sufficiently large. As a result the integral in Eq. 4 does not vanish because one actually has to integrate only over these fractions of the total period when the derivative $\dot{\mathrm{E}}$ is large enough. More detailed equations which describe the layer rotation in the $\mathrm{SmC}^{*}$ phase are described in the following subsection which is based on the results obtained by Carlsson and Osipov in [111].

\subsection{General equations of motion for the layer rotation: Thermodynamic} approach

Let us consider a SmA* liquid crystal in the bookshelf geometry with an external time-dependent electric field applied in a direction parallel to the smectic planes. It is well known that due to the electroclinic effect, the director $\mathbf{n}$ will deviate from the layer normal, and this deviation is specified by the tilt angle $\Theta$ which is the first natural dynamic variable of this system. The second dynamic variable is the angle $\gamma$ between the 
layer normal $\mathbf{k}$ and the axes of the fixed coordinate system. The absolute value of the angular velocity of layer rotation is then equal to the time derivative of $\gamma$.

One notes that the two dynamic variables in the system i.e., the tilt angle $\Theta$ and the layer rotation angle $\gamma$ are not equivalent from a thermodynamic point of view. Indeed, the free energy of the $\mathrm{SmC}^{*}$ phase depends on $\Theta$ but is independent of $\gamma$. The dynamics of $\Theta$ is relaxational in nature, i.e., the tilt angle relaxes to its equilibrium value determined by the field. On the other hand, the change of $\gamma$ is also associated with some energy dissipation. In this sense the angle $\Theta$ is analogous to the director $\mathbf{n}$ in the homogeneous nematic phase. In the latter case the free-energy density also does not depend on the orientation of the director, but the rotation of $\mathbf{n}$ is accompanied by a dissipation. In the SmA* phase subjected to an external electric field, the variables $\Theta$ and $\gamma$ are coupled, and we will see that this coupling determines the rotation of the smectic layers.

The dynamics of such a system can be described by the general approach based on the Rayleigh dissipation function. The application of this approach to the study of the dynamics of nematic liquid crystals is discussed in detail by Vertogen and de Jeu [108]. In this approach the state of the system which is assumed to be close to thermodynamic equilibrium is specified by a set of macroscopic variables $X_{i}=(\Theta, \gamma)$ and their time derivatives $X_{i}=(\Theta, \gamma) \quad$. The properties of the system are described by the free-energy density functional $F\left(X_{i}\right)$ and the dissipation function $D\left(X_{1}\right)$. Then the dynamics of the system is described by a set of Euler-Lagrange equations:

$$
\frac{\partial D}{\partial \dot{\gamma}}=\frac{\partial F}{\partial \gamma}
$$




$$
\frac{\partial D}{\partial \dot{\Theta}}=\frac{\partial F}{\partial \Theta}
$$

In the general case, the dissipation function $\mathrm{D}$ can be expressed as a quadratic form of the time derivatives $\cdot \dot{\Theta}$ and $\cdot \dot{\gamma}$

$$
D=\frac{1}{2} \gamma_{1} \dot{\Theta}^{2}+\frac{1}{2} \lambda \dot{\gamma^{2}}+\frac{1}{2} \gamma_{12} \dot{\gamma} \dot{\Theta}
$$

where $\gamma_{1}$ is the rotational viscosity of the $\mathrm{SmC}^{*}$ phase, $\lambda$ is the rotational viscosity associated with the layer rotation and where the last term describes the dynamic coupling between the variables $\gamma$ and $\Theta$.

Here, the free energy of the $\mathrm{SmC}^{*}$ phase is given by the well known equation ( see, for example, $[109,110])$

$$
F=\frac{1}{2} a(T) \Theta^{2}+\frac{1}{2 \varepsilon} P^{2}-C P \Theta-P E,
$$

where $P$ is the spontaneous polarization, $\varepsilon$ is the transverse dielectric constant and $C$ is the pseudo-scalar coupling constant.

Minimization of the free energy with respect to $\Theta$ and $P$ yields the following equations:

$$
\begin{gathered}
\frac{\partial F}{\partial \Theta}=a(T) \Theta-C P=0, \\
\frac{\partial F}{\partial P}=\frac{1}{\varepsilon} P-C \Theta-E=0 .
\end{gathered}
$$


Expressing $P$ from the second equation and substituting it into the first, one obtains the following expression for the derivative $\partial F / \partial \Theta$ which enters one of the Euler-Lagrange equations:

$$
\frac{\partial F}{\partial \Theta}=a(T) \Theta-\varepsilon C E
$$

One notes also that $\partial F / \partial \gamma=0$ because the free energy $F$ does not depend on $\gamma$. Using these results, eqs. (4) can be rewritten in the simple form:

$$
\begin{aligned}
& \gamma_{1} \dot{\gamma}+\gamma_{12} \dot{\Theta}=0 \\
& \gamma_{1} \dot{\Theta}+\gamma_{12} \dot{\gamma}=-a(T) \Theta+\varepsilon C E
\end{aligned}
$$

Here the first equation describes a relaxation of the tilt angle in a rotating layer while the second equation represents a counterbalance of the two generalized torques acting on the smectic layer normal.

One notes further, that there are three different time scales involved in Eqs (9). . The response time of the electroclinic effect $\tau_{r}=a(T) / \gamma_{1}$ falls within the submillisecond regime according to the experimental measurements [64Error! Reference source not found.]. At the same time the rotation of the smectic layers has been found experimentally to correspond to a time scale of several seconds. Thus the term $\gamma_{12} \dot{\gamma}$ in Eq.(9.) can be neglected and the equation can be approximated as

$$
\gamma_{1} \dot{\Theta}=-a(T) \Theta+\varepsilon C E
$$

This is the equation governing the dynamic behavior of the electroclinic effect in the system with fixed smectic layers. 
The solution of eq.(10) can be substituted in to eq.(9) to obtain $\dot{\gamma}$ :

$$
\dot{\gamma}=\kappa \dot{\Theta}
$$

where $\kappa=\lambda_{12} / \lambda_{1}$.

In the next section we obtain explicit expressions for the angle of layer rotation using the particular form of the saw-tooth external electric field.

\subsection{Rotation of the smectic layers caused by an asymmetric electric field}

The time variation of the asymmetric saw-tooth electric field is presented in Fig. 17 . The field is assumed to be oscillating between the values $+E_{0}$ and $-E$ ). The rise time $\tau_{1}$ is assumed to be shorter than the decay time $\tau_{2}$, and the asymmetry ratio of the field is introduced as $h=\tau_{1} / \tau_{2}$. The frequency of the field is $f=\left(\tau_{1}+\tau_{2}\right)^{-1}$. Mathematically, the variation of the electric field within one period can be written in the form:

$$
E(t)=E_{0}\left(\frac{2 t}{\tau_{1}}-1\right)
$$

when $0<t<\tau_{1}$ and

$$
E(t)=E_{0}\left(\frac{2 \tau_{1}}{\tau_{2}}+1-\frac{2 t}{\tau_{2}}\right)
$$

when $\tau_{1}<t<\tau_{2}$ 
Due to the fact that the rate of change of the electric field is much slower than the electroclinic response time, one can safely assume that during the switching the tilt will always adopt its equilibrium value for the given electric field. The rate of change of the tilt is then simply given by

$$
\dot{\Theta}=\frac{\varepsilon C}{a_{0}} E
$$

The angular velocity of layer rotation is now expressed as

$$
\dot{\gamma}=\sigma E
$$

where $\sigma=\kappa \varepsilon C / a_{0}$. Using the explicit expression for the saw-tooth electric field one obtains

$$
\dot{\gamma}=\frac{2 \sigma}{\tau_{1}} E
$$

when $0<t<\tau_{1}$ and

$$
\dot{\gamma}=-\frac{2 \sigma}{\tau_{2}} E
$$

when $\tau_{1}<t<\tau_{2}$.

The net rotation, $\Delta \gamma$, of the smectic layers during one period of the electric field is now calculated as

$$
\Delta \gamma=\int_{0}^{\tau_{1}+\tau_{2}} \gamma d t .
$$


The value of $\Delta \gamma$ calculated from Eqs.(16-18) is obviously zero because the larger positive value of $\gamma$ during the shorter time $\tau_{1}$ is exactly compensated by the smaller negative value of $\gamma$ during the longer time interval $\tau_{2}$.

However, there must exist a stabilizing torque $\Gamma_{S}$ which is related to the surface treatment of the glass plates surrounding the sample, and which is responsible for keeping the preferred orientation of the layers. In order for the layers to rotate, the driving torque must exceed some threshold, corresponding to the maximum possible value of the stabilizing torque $\Gamma_{0}$. The stabilizing torque $\Gamma_{S}$ has the nature of a friction torque and adopts the value needed to balance the driving torque as long as it is smaller then $\Gamma_{0}$. However, if the driving torque exceeds a critical value, the stabilizing torque can no longer increase, but adopts its maximum value $\Gamma_{0}$, always opposing the rotation of the layers. By adding the stabilizing torque with the proper sign to Eqs., , one obtains the final dynamic equations for the layer normal:

$$
\dot{\gamma}=\frac{2 \sigma}{\tau_{1}} E-\frac{2 \Gamma_{0}}{\gamma_{1} d}
$$

if $\quad E_{0}>\frac{\Gamma_{0} \tau_{1}}{d \gamma_{1} \sigma}$,

and

$$
\dot{\gamma}=0
$$

if $E_{0}<\frac{\Gamma_{0} \tau_{1}}{d \gamma_{1} \sigma}$ when $0<t<\tau_{1}$. 
Similarly,

$$
\dot{\gamma}=-\frac{2 \sigma}{\tau_{2}} E+\frac{2 \Gamma_{0}}{\gamma_{1} d}
$$

if $E_{0}>\frac{\Gamma_{0} \tau_{1}}{d \gamma_{1} \sigma}$ and

$$
\dot{\gamma}=0
$$

if $E_{0}<\frac{\Gamma_{0} \tau_{2}}{d \gamma_{1} \sigma}$ when $\tau_{1}<t<\tau_{2}$. Here we have taken into account that $\Gamma_{0}$ is the surface torque,

that is, the torque per unit area.

Eqs. $(19,21)$ imply that there exist two threshold values of the electric field:

and

$$
E_{1}=\frac{\Gamma_{0} \tau_{1}}{d \gamma_{1} \sigma}
$$

$$
E_{2}=\frac{\Gamma_{0} \tau_{2}}{d \gamma_{1} \sigma}
$$

Note that $E_{1}<E_{2}$ when $\tau_{1}<\tau_{2}$.

The average angular velocity $\omega=<\dot{\gamma}>$ of the layers is then obtained as

$$
\omega=f \Delta \gamma
$$

where $f$ is the frequency of the applied field and $\Delta \gamma$ the net rotation during one period of the electric field. Using Eqs.(19-22) the average angular velocity of the smectic layer rotation as a function of the amplitude of the electric field $E_{0}$ can be expressed as: 


$$
\omega=0
$$

if $E_{0}<E_{1}$,

$$
\omega=\frac{2 f \Gamma_{0} \tau_{1}}{d \gamma_{1}}\left(\frac{E_{0}}{E_{1}}-1\right),
$$

if $E_{1}<E_{0}<E_{2}$ and

$$
\omega=\frac{2 f \Gamma_{0}}{d \gamma_{1}}\left(\tau_{2}-\tau_{1}\right)
$$

if $E_{2}<E_{0}$.

These final equations can also be rewritten in the following non dimensional form [111]: $\omega=0$ if $E_{0}^{*}<E_{1}^{*}$,

$$
\omega^{*}=2 \kappa\left[f^{*} E_{0}^{*}-1 / d^{*}(1+\eta)\right]
$$

if $E_{1}^{*}<E_{0}^{*}<E_{2}^{*}$ and

$$
\omega^{*}=2 \kappa \frac{\eta-1}{d(1+\eta)}
$$

if. $E_{2}^{*}<E_{0}^{*}$.

Here $E^{*}=\delta C E / a_{0}, d^{*}=d a_{0} \lambda_{12} / \lambda_{2} \Gamma_{0} . f^{*}=f \lambda_{2} / a_{0}$ and $\eta=\tau_{1} / \tau_{2}$. One notes that these non dimensional equations contain only three independent control parameters including the product of the frequency and the strength of the electric field $f^{*} E_{0}^{*}$, the asymmetry ratio $\eta$, and the sample thickness $d^{*}$. It is also seen that only one combination of the material constants enters the final equations. This is the ratio $. \kappa=\lambda_{12} / \lambda_{1}$. 
The dependence of the average angular velocity of smectic layer rotation is presented in Fig. 17 as a function of the product $f^{*} E_{0}^{*}$ for four different values of the asymmetry ratio $\eta$. One can readily see that there exists a lower value of $f^{*} E_{0}^{*}$ below which there is no layer rotation, corresponding to the threshold given by Eq. (23) One can also see from the figure that the threshold decreases with the increasing asymmetry $\eta$. With the increasing $f^{*} E_{0}^{*}$ above the threshold the angular velocity increases linearly until the second threshold (determined by Eq.(23b)) is reached and the angular velocity becomes saturated, independent of. $f^{*} E_{0}^{*}$.

The average angular velocity of the layers divided by $\kappa$ is plotted as a function of the sample thickness in Fig.18 One notes that the smectic layers rotate only if the layer thickness exceeds some threshold value $d_{t r}^{*}$ which depends on $\eta$. Such a threshold has indeed been observed experimentally. It is interesting to note also that there exists an optimum sample thickness which corresponds to the largest possible angular velocity of layer rotation for given $\eta$ and. $f^{*} E_{0}^{*}$ This behavior can be explained qualitatively in the following way. Once the threshold $d_{t r}^{*}$ is exceeded, the average angular velocity increases with the increasing sample thickness. This is due to the fact that the driving torque is a bulk effect which increases with increasing sample thickness. On the other hand, the counter-torque, acting only at the surfaces of the substrates, is independent of the sample thickness. As long as the second threshold (see $\operatorname{Eq}(23 b)$ ) is not exceeded, the layers rotate only during the interval $\tau_{1}$ of the total period of the electric field. At a certain value of the sample thickness, however, the second threshold is exceeded and the layers rotate in opposite directions during the intervals $\tau_{1}$ and $\tau_{2}$ within the same period. 
In this regime it is the difference between the driving torque and the counter-torque that is responsible for the net rotation of the layers. The relative difference between these torques during the intervals $\tau_{1}$ and $\tau_{2}$ decreases with increasing sample thickness. Thus,

for a very thick sample the layers just oscillate back and forth, and the average rotation of the layers approaches zero.

\section{Summary}

This review summarizes the observation and behaviour of smectic layer rotational instabilities, or short, the smectic layer rotation, which can be observed by application of an asymmetric electric field to a chiral fluid smectic liquid crystal. Textures and small angle x-ray diffraction are used to elucidate the respective structures. The dependence of the layer rotation on a variety of parameters is detailed, such as the field asymmetry ratio, the applied field frequency and bias, the temperature approaching the $\mathrm{SmC}^{*}-\mathrm{SmA}^{*}$ transition (thus the influence of the electroclinic effect), or the cell gap. Also other parameters are discussed, like the enantiomeric excess, varying alignment layers (thus indirectly the anchoring strength), the concentration of ions and the phase sequence of a specific material.

Since the smectic layer instabilities have a detrimental effect on most of the applications of ferroelectric liquid crystals, some ways for their avoidance, like polymer stabilization, are discussed, and even applications based on the layer rotation are proposed, such as optical recording. At last, a comprehensive theoretical description is developed, which accounts for all of the observations described. 


\section{References}

[1] M. Mitov, Chem. Phys. Chem., 2014, 15, 1245.

[2] P.J. Collings, M. Hird, Introduction to Liquid Crystals: Chemistry and Physics, Taylor\&Francis, London, 1997.

[3] S. Chandrasekhar, Liquid Crystals, $2^{\text {nd }}$ ed., Cambridge University Press, Cambridge, 1992.

[4] P.G. de Gennes, J. Prost, The Physics of Liquid Crystals, 2nd ed., Clarendon Press, Oxford, 1993.

[5] D. Demus, J. Goodby, G.W. Gray, H.-W. Spiess, V. Vill (eds.), Physical Properties of Liquid Crystals, Wiley-VCH, Weinheim, 1999.

[6] D. Demus, J. Goodby, G.W. Gray, H.-W. Spiess, V. Vill (eds.), Handbook of Liquid Crystals, Wiley-VCH, Weinheim, 1998.

[7] L.M. Blinov, V.G. Chigrinov, Electrooptic Effects in Liquid Crystal Materials, Springer Verlag, New York, 1994.

[8] E. Lueder, Liquid Crystal Displays, John Wiley \& Sons, Chichester, 2001.

[9] S.-T. Wu, D.-K. Yang, Reflective Liquid Crystal Displays, John Wiley \& Sons, Chichester, 2001.

[10] Wuan Li, Liquid Crystals Beyond Displays, Wiley \& Sons, Hoboken, NJ, 2012.

[11] I.W. Hamley, Introduction to Soft Matter, Wiley \& Sons, Chichester, 2000.

[12] M. Kleman, O.D. Lavrentovich, Soft Matter Physics, Springer, New York, 2003.

[13] H.-S. Kitzerow, C. Bahr (eds.), Chirality in Liquid Crystals, Springer, New York, 2001. 
[14] S.T. Lagerwall, Ferroelectric and Antiferroelectric Liquid Crystals, Wiley-VCH, Weinheim, 1999.

[15] I. Musevic, R. Blinc. B. Zeks, The Physics of Ferroelectric and Antiferroelectric Liquid Crystals, World Scientific, Singapore, 2000

[16] A. Buka, L. Kramer (eds.), Pattern Formation in Liquid Crystals, Springer, New York, 1996.

[17] G.P. Crawford, S. Zumer (eds.), Liquid Crystals in Complex Geometries, Taylor \& Francis, London, 1996.

[18] I. Dierking, Adv. Mater., 2000, 12, 167.

[19] I. Dierking, Materials, 2014, 7, 3568

[20] I. Dierking in, Chemistry of Carbon Nanotubnes, Vol.2, chapter 10, V.A. Basiuk, E.V. Basiuk (eds.), American Scientific Publishers, Stevenson Range, USA, 2008

[21] M. Mitov, Adv. Mater., 2012, 24, 6260.

[22] M. Kleman, O.D. Lavrentovich, Phil. Mag., 2006, 86, 4117

[23] I. Dierking, Textures of Liquid Crystals, Wiley-VCH, Weinheim, 2003.

[24] A.C. Neville, Biology of Fibrous Composites-Development Beyond the Cell Membrane, Cambridge University Press, 1993.

[25] Structural Biological Materials-Design and Structure-Property Relationship (Ed.: M. Elices) Pergamon, 2000.

[26] Y. Bouligand, Ann. Chim. Sci. Mat. 2004, 29, 83-96.

[27] Y. Bouligand, C. R. Acad. Chimie 2008, 11, 212-220.

[28] A.M. Figueiredo Neto, S.R.A. Salinas, The Physics of Lyotropic Liquid Crystals, Oxford University Press, Oxford, 2005. 
[29] I. Dierking, Symmetry, 2014, 6, 444

[30] R.B. Meyer, L. Liebert, L. Strzelecki, P. Keller, J. Phys. Lett. (Fr.), 1975, 36, L69.

[31] S. Garoff, R.B. Meyer, Phys. Rev. Lett., 1977, 38, 848.

[32] S. Garoff, R.B. Meyer, Phys. Rev. A, 1979, 19, 338.

[33] P. Martinot-Lagarde, J. de Phys., 1976, 37, C3-129.

[34] G. Durand, P. Martinot-Lagarde, Ferroelectrics, 1980, 24, 89.

[35] N.A. Clark, S.T. Lagerwall, Appl. Phys. Lett., 1980, 36, 899.

[36] T.P. Rieker, N.A. Clark, G.S. Smith, D.S. Parma, E.B. Sirota, C.R. Safinya, Phys. Rev. Lett., 1987, 59, 2658.

[37] N.A. Clark, T.P. Rieker, Phys. Rev. A, 1988, 37, 1053.

[38] T.P. Rieker, N.A. Clark, G.S. Smith, C.R. Safinya, Liq. Cryst., 1989, 6, 565.

[39] Y. Ouchi, J. Lee, H. Takezoe, A. Fukuda, K. Kondo, T. Kitamura, A. Mukoh, Jpn. J. Appl. Phys., 1989, 27, L725.

[40] M. Johno, A.D.L. Chandani, Y. Ouchi, H. Takezoe, A. Fukuda, M. Ichihashi, K. Furukawa, Jpn. J. Appl. Phys., 1989, 28, L119.

[41] K. Itoh, M. Johno, Y. Takanishi, Y. Ouchi, H. Takezoe, A. Fukuda, Jpn. J. Appl. Phys., 1991, 30, 735.

[42] M. Oh-E, M. Isogai, T. Kitamura, Liq. Cryst., 1992, 11, 101.

[43] F. Giesselmann, P. Zugenmaier, Mol. Cryst. Liq. Cryst., 1993, 237, 121.

[44] R.A.M. Hikmet, Liq. Cryst., 1995, 18, 927

[45] A.S. Morse, H.F. Gleeson, S. Cummings, Liq. Cryst., 1997, 23, 717 
[46] L.A. Parry-Jones, S.M. Beldon, P.D. Brimicombe, R.M. Richardson, D. Rodriguez-Martin, S.J. Elston, J.G. Grossmann, G.R. Mant, Liq. Cryst., 2006, 33, 767

[47] A. Jakli, A. Saupe, Phys. Rev. A, 1992, 45, 5674.

[48] L. Lejcek, S. Pirkl, Liq. Cryst., 1990, 8, 871.

[49] J. Pavel, M. Glogarova, Liq. Cryst., 1991, 9, 87.

[50] R. Shao, P.C. Willis, N.A. Clark, Ferroelectrics, 1991, 121, 127.

[51] K. Skarp, G. Andersson, T. Hirai, A. Yoshizawa, K. Hiraoka, H. Takezoe, A. Fukuda, Jpn. J. Appl. Phys., 1992, 31, 1409.

[52] S.-D. Lee, J.-H. Kim, J.S. Patel, J.W. Goodby, Mol. Cryst. Liq. Cryst., 1993, 227, 39.

[53] Y. Asao, T. Uchida, Jpn. J. Appl. Phys., 1993, 32, L604.

[54] G. Andersson, K. Flatischler, L. Komitov, S.T. Lagerwall, K. Skarp, B. Stebler, Ferroelectrics, 1991, 113, 361.

[55] I. Dierking, F. Giesselmann, J. Schacht, P. Zugenmaier, Liq. Cryst., 1995, 19, 179

[56] I. Dierking, L. Komitov, S.T. Lagerwall, Liq. Cryst., 1998, 24, 769

[57] I. Dierking, B. Glüsen, S.T. Lagerwall, C.K. Ober, Phys. Rev. E, 2000, 61, 1593

[58] S. Shibahara, J. Yamamoto, Y. Takanishi, K. Ishikawa, H. Takezoe, J. Phys. Soc. Jap., 2002, 71, 802

[59] G. Carbone, B. Zappone, R. Barberi, R. Bartolino, I. Musevic, Phys. Rev. E, 2011, 83, 051707

[60] G. Andersson, I. Dahl, L. Komitov, S.T. Lagerwall, M. Matuszczyk, K. Skarp, presented at the 13th International Liquid Crystal Conference, Vancouver, Canada, 1990 
[61] K. Skarp, G. Andersson, T. Hirai, A. Yoshizawa, K. Hiraoka, H. Takezoe, A. Fukuda, presented at the 14th International Liquid Crystal Conference, Pisa, Italy, 1992

[62] G. Andersson, T. Carlsson, S.T. Lagerwall, M. Matuszczyk, T. Matuszczyk, presented at the 4th International Conference on Ferroelectric Liquid Crystals, Tokyo, Japan, 1993

[63] K. Nakayama, M. Ozaki, K. Yoshino, Jpn. J. Appl. Phys., 1996, 35, 6200

[64] K. Nakayama, H. Moritake, M. Ozaki, K. Yoshino, Jpn. J. Appl. Phys., 1995, 34, L1599

[65] S.V. Yablonskii, K. Nakayama, M. Ozaki, K. Yoshino, JETP Lett., 1998, 67, 978

[66] M. Ozaki, H. Moritake, K. Nakayama, K. Yoshino, Jpn. J. Appl. Phys., 1994, 33, L1620

[67] H. Moritake, K. Nakayama, M. Ozaki, K. Yoshino, Ferroelectrics, 1996, 178, 277

[68] I. Dierking, L. Komitov, S.T. Lagerwall, Jpn. J. Appl. Phys., 1998, 37, L57

[69] K. Nakayama, M. Ozaki, K. Yoshino, Appl. Phys. Lett., 1997, 70, 2117

[70] I. Dierking, L. Komitov, S.T. Lagerwall, Jpn. J. Appl. Phys., 1998, 37, L525

[71] Chang-Jae Yu, Wan-Soo Kim, Sin-Doo Lee, Ferroelectrics, 2004, 311, 77

[72] I. Dierking, Ferroelectrics, 1999, 234, 171

[73] I. Dierking, F. Giesselmann, J. Kusserow, P. Zugenmaier, Liq. Cryst., 1994, 17, 243

[74] I. Dierking, S.T. Lagerwall, Ferroelectrics, 1999, 227, 97

[75] I. Dierking, L. Komitov, S.T. Lagerwall, Ferroelectrics, 1998, 215, 11

[76] I. Dierking, L. Komitov, S.T. Lagerwall, Ferroelectrics, 1998, 211, 165

[77] I. Dierking, L. Komitov, S.T. Lagerwall, Ferroelectrics, 1998, 211, 259 
[78] I. Dierking, L. Komitov, S.T. Lagerwall, Liq. Cryst., 1998, 24, 775

[79] K. Nakayama, M. Ozaki, K. Yoshino, Jpn. J. Appl. Phys., 1998, 37, 5379

[80] K. Nakayama, J. Ohtsubo, M. Ozaki, K. Yoshino, Appl. Phys. Lett., 2002, 80, 2439

[81] K. Nakayama, J. Ohtsubo, M. Ozaki, K. Yoshino, Mol. Cryst. Liq. Cryst., 2005, 434, 87

[82] K. Nakayama, J. Ohtsubo, M. Ozaki, K. Yoshino, Mol. Cryst. Liq. Cryst., 2004, 409, 243

[83] R. A. M. Hikmet, Liq. Cryst., 9, 405 (1991).

[84] I. Dierking, L. L. Kosbar, A. Afzali-Ardakani, A. C. Lowe, G. A. Held, J. Appl. Phys., 1997, 81, 3007

[85] M. Mitov, A. Boudet, P. Sopena, P. Sixou, Liq. Cryst., 1997, 23, 903

[86] J. Pirs, R. Blinc, B. Marin, S. Pirs, J. W. Doane, Mol. Cryst. Liq. Cryst., 1995, 264, 155

[87] R. A. M. Hikmet, H. M. J. Boots, M. Michielsen, Liq. Cryst., 1995, 19, 65

[88] R. A. M. Hikmet, J. Appl. Phys., 1990, 68, 4406

[89] D.-K. Yang, L.-C. Chien, J. W. Doane, Appl. Phys. Lett., 1992, 60, 3102

[90] M. Mitov, N. Dessaud, Nature Mater., 2006, 5, 361

[91] H. Ishii-Erikson, MSc thesis, Chalmers Univ. of Technology, Göteborg (Sweden), 1995.

[92] M. Mitov, H. Ishii, S. T. Lagerwall, P. Sixou, G. Andersson and L. Komitov, poster E2.O05, 16 ${ }^{\text {th }}$ Int. Liquid Crystal Conf., Kent (USA), 1996.

[93] M. Mitov, P. Sixou, Rev. Scientif. Tech. Défense, 1997, 2, 239. 
[94] I. Dierking, L. Komitov, S. T. Lagerwall, T. Wittig, R. Zentel, Liq. Cryst., 1999, 26, 1511

[95] I. Dierking, thesis, Chalmers Univ. of Technology, Göteborg (Sweden), 1999.

[96] P. Archer, I. Dierking, M. Osipov, Phys. Rev. E, 2008, 78, 051703

[97] P. Archer, I. Dierking, J. Phys. D: Appl. Phys., 2008, 41, 155422

[98] C. A. Guymon, E. N. Hoggan, D. M. Walba, N. A. Clark, C. N. Bowman, Liq. Cryst., 1995, 19, 719

[99] C. A. Guymon, L. A. Dougan, P. J. Martens, N. A. Clark, D. M. Walba, C. N. Bowman, Chem. Mater., 1998, 10, 2378

[100] C. A. Guymon, E. N. Hoggan, N. A. Clark, Science, 1997, 275, 57

[101] J. Nourry, P. Sixou, M. Mitov, M. Glogarova, A. M. Bubnov, Liq. Cryst., 2000, 27, 35

[102] J. Nourry, A. Vigouroux, A. Magnaldo, P. Sixou, M. Mitov, A. Boudet, M. Glogarova, A. M. Bubnov, Ferroelectrics, 1998, 212, 203

[103] S. Suresh, L.-C. Chien, Ferroelectrics, 2003, 287, 1

[104] I. Dierking, M. A. Osipov, S. T. Lagerwall, Eur. Phys. J. E, 2000, 2, 303

[105] M. Gasser, A. Gembus, D. Ganzke, I. Dierking, Mol. Mater., 2000, 12, 347

[106] J. Pirs, P. Bos, R. Petkovsek, S. Kralj, S . Pirs, S. Zumer, T. Matuszczyk, Jpn. J. Appl. Phys., 2002, 41, 6011

[107] P. Archer, I. Dierking, J. Opt. A: Pure Appl. Opt., 2009, 11, 024022

[108] G. Vertogen and W. H. de Jeu, Thermotropic Liquid Crystals,Fundamentals, Springer Series in Chemical Physics Vol. 45 Springer, Berlin, 1988, pp. 140-148

[109] T. Carlsson, F. M. Leslie, and N. A. Clark, Phys. Rev. E 51, 4509 1995. 
[110] L. A. Beresnev, L. M. Blinov, M. A. Osipov, S. A. Pikin. Ferroelectric Liquid Crystals Mol. Cryst. Liq. Cryst. Special Topics XXIX 158 A, 1 - 150, (1988).

[111]. T.Carlsson and M.A.Osipov, Phys. Rev. E, 60, 5619 (1999). 


\section{Figure captions}

Figure 1: Schematic scheme of the different states of matter (top). The liquid crystal states are observed between the three dimensionally ordered crystal and the (isotropic) liquid phase. Within the temperature interval of the liquid crystal state, several phases can be observed, which differ in their degree of order.

Figure 2: Schematic illustration of the symmetry elements of the achiral smectic $\mathrm{C}$ phase. If the phase consists of chiral molecules, the mirror plane and the inversion center vanish, and a net dipole moment along the rotation axis is observed, which constitutes the spontaneous polarization of the ferroelectric smectic $\mathrm{C}^{*}$ phase. (Reproduced by permission from [23]. Copyright Wiley-VCH).

Figure 3: (a) schematic representation of the helical superstructure of the smectic $C^{*}$ phase, due to a local cancellation of the spontaneous polarization, Ps. (b) Under appropriate boundary conditions, the signature of the periodic helicoidal structure appears by polarizing microscopy as a (periodic) stripe texture. The scale bar is approximately $25 \mu \mathrm{m}$.

Figure 4: (a) in the bookshelf geometry the smectic layers are formed perpendicular to the substrate plates, with two possible positions of the spontaneous polarization, up or down. The latter are related with two positions of the director on the tilt cone. (b) in a surface stabilized ferroelectric liquid crystal the two director positions can be visualized by 
polarizing microscopy as two types of domains, dark and bright. The scale bar is approximately $25 \mu \mathrm{m}$.

Figure 5: (a) schematic of a vertical chevron device with the smectic layers being tilted with respect to the substrate plane by an angle, which is often about equal to the director tilt angle, $\delta \sim \theta$. (b) vertical chevrons can exist in two tilt directions. A defect wall (thick or thin) is observed where chevrons of different tilt direction meet. (c) In polarizing microscopy these defect walls are seen to make a zig-zag defect. The scale bar is

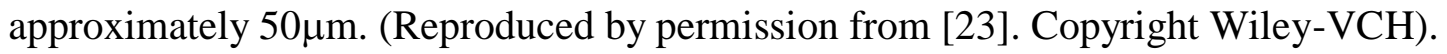

Figure 6: (a) in the chiral smectic $A^{*}$ phase layer straightening by application of a sufficiently large electric field can lead to stripe structure, where the contrast between different stripes depends on the induced tilt angle. (b) Demonstration of the stripe texture in polarizing microscopy, where the width of the individual stripes is often observed to be equal to the sandwich cell gap d. (Reproduced by permission from [23]. Copyright Wiley-VCH).

Figure 7: (a) model of a zig-zag defect in a vertical chevron structure. The regions of opposite chevron direction are of the bookshelf type. (b) model of a horizontal chevron domain structure, as can be observed after application of a large electric field to a vertical chevron structure. The defect mediating the horizontal chevron domains of opposite inclination is of the vertical chevron type. (c) polarizing microscopic example of a horizontal chevron structure. The smectic layers can easily be visualized through the 
equidistant helical line pattern. (Reproduced by permission from [55]. Copyright Taylor\&Francis).

Figure 8: Switching process of a horizontal domain texture (left), and respective schematic illustrating the director directions (right). (a) zero field, (b) positive field and (c) negative field. Note that on polarity reversal bright and dark domains are interchanged. The bottom of the figure illustrates the formation of horizontal chevrons by time resolved synchrotron small angle $\mathrm{x}$-ray scattering from an unoriented powder sample to the horizontal chevron structure exhibiting two domain types ( $t=0 \mathrm{~s}-20 \mathrm{~s}$, left to right). (Top: Reproduced by permission from [23]. Copyright Wiley-VCH. Bottom: Reproduced by permission from [57]. Copyright American Physical Society).

Figure 9: Electro-optic response of a horizontal chevron structure. The two individual domains (dotted and dash-dotted lines) exhibit standard electro-optic FLC switching, though phase shifted by 180 degrees. The detrimental effect of the horizontal chevrons on the electro-optic response can be seen for the switching of the whole cell (dashed line), where the transmission is hardly modulated at all. (Reproduced by permission from I. Dierking, G. Scalia, Liq. Cryst. 27, (2000), 1059. Copyright Taylor\&Francis).

Figure 10: Formation time $t_{r}$ and average domain width $W_{d}$ of horizontal chevron structures formed as a function of (a) electric field amplitude E, (b) field frequency $f$ and reduced temperature $\mathrm{T}-\mathrm{T}_{\mathrm{C}}$ with respect to the $\mathrm{SmA}^{*}{ }_{-} \mathrm{SmC} \mathrm{C}^{*}$ transition. 
Figure 11: Rotational smectic layer instabilities observed after rotating of the layers by several hundreds of degrees. Courtesy of Gunnar Andersson, Department of Physics, Chalmers University of Technology, Gothenburg, Sweden. The scale bar is approximately $100 \mu \mathrm{m}$. (Reproduced by permission from [23]. Copyright Wiley-VCH).

Figure 12: Dependence of horizontal chevron reorientation times on applied external conditions of (a) electric field amplitude, (b) field frequency, (c) reduced temperature $\left(\mathrm{T}_{\mathrm{c}}\right.$ is the $\mathrm{SmC}^{*}-\mathrm{SmA} \mathrm{A}^{*}$ transition temperature), and (d) cell gap. Experimental conditions are noted in the figure.

Figure 13: Smectic layer permeation model for the domain wall motion of horizontal chevrons during the reorientation process from one to the other domain type of opposite layer inclination (top left). Only a small molecular displacement is needed for the rotation to be accomplished. Exemplary series of textures demonstrating the permeation reorientation model through the deformation of circular air bubbles (top right). The bottom row shows respective time resolved small angle synchrotron x-ray images of the reorientation process ( $\mathrm{t}=0 \mathrm{~s}-50 \mathrm{~s}$ from (a) to (d)). (Top: Reproduced by permission from [74]. Copyright Taylor\&Francis. Bottom: Reproduced by permission from [57]. Copyright American Physical Society).

Figure 14: Dependence of the layer reorientation time on several materials conditions as a function of electric field asymmetry. (a) variation of enantiomeric excess, (b) different alignment layers with varying anchoring strength, (c) ionic content, and (d) variation of 
phase sequence within a homologous series of FLC compounds from $\mathrm{N}^{*}-\mathrm{SmC}^{*}$ via $\mathrm{N}^{*}$ TGBA*-SmC* to SmA*-SmC*.

Figure 15: Optical recording by use of the smectic layer reorientation. The top images show a schematic of the process with (a) and (b) the recording and (c) and (d) the erasing cycle. The bottom images show a respective experimental verification with (a) recording and (b) erasing. (Reproduced by permission from [80]. Copyright AIP Publishing).

Figure 16: optical textures of a polymer-stabilized ferroelectric liquid crystal: (a) at 0 min. (left) and after 10 min. under an asymmetric field (right) in the $\mathrm{SmA}^{*}$ phase. The smectic layer rotation is suppressed; (b) birefringent patterns in the stripe texture remain visible although most of the liquid crystal has undergone the transition to the isotropic state.

Figure 17. Average nondimensional angular velocity of the smectic layers divided by $\kappa$ as a function of the product between the frequency and the amplitude of the electric field. for four different values of the asymmetry ratio $\eta=\tau_{1} / \tau_{2}$. Here the nondimensional sample thickness $d^{*}$ is . $d^{*}=100$.

Figure 18. Average nondimensional angular velocity of the smectic layers divided by $\kappa$ as a function of the nondimensional sample thickness $d^{*}$ for four different values of the asymmetry ratio $\eta=\tau_{1} / \tau_{2}$ using the fixed value $f^{*} E_{0}^{*}=0.005$ 
Figure 1 
Figure 2 
Figure 3 
Figure 4 
Figure 5 
Figure 6 
Figure 7 
Figure 8 
Figure 9 
Figure 10

Figure 11 
Figure 12: 
Figure 13 
Figure 14 
Figure 15 
(a)

(b)

Figure 16 